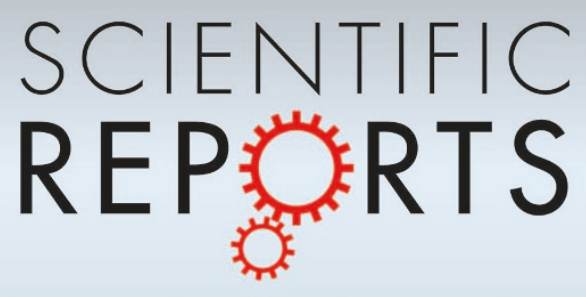

OPEN

SUBJECT AREAS:

CHEMICAL PHYSICS

ELECTRONIC PROPERTIES AND

DEVICES

Received

21 August 2014

Accepted

14 November 2014

Published

3 December 2014

Correspondence and requests for materials should be addressed to

Y.D. (daiy60@sina. com)

\section{Strain-induced quantum spin Hall effect in methyl-substituted germanane $\mathrm{GeCH}_{3}$}

\author{
Yandong Ma', Ying Dai', Wei Wei', Baibiao Huang' \& Myung-Hwan Whangbo²
}

'School of Physics, State Key Laboratory of Crystal Materials, Shandong University, Jinan 250100, People's Republic of China,
2Department of Chemistry, North Carolina State University, Raleigh, North Carolina 27695-8205, USA.

Quantum spin Hall (QSH) insulators exhibit a bulk insulting gap and metallic edge states characterized by nontrivial topology. We investigated the electronic structure of an isolated layer of methyl substituted germanane $\mathrm{GeCH}_{3}$ by density functional calculations (DFT), and its dynamic stability by phonon dispersion calculations. Our results show that an isolated $\mathrm{GeCH}_{3}$ layer has no dynamic instability, and is a QSH insulator under reasonable strain. This QSH insulator has a large enough band gap (up to $108 \mathrm{meV}$ ) at $12 \%$ strain. The advantageous features of this QSH insulator for practical room-temperature applications are discussed.

ecently, two-dimensional (2D) topological insulators (TIs), namely, QSH insulators ${ }^{1-7}$, have attracted much attention. These insulators possess a bulk insulting gap but metallic edge states. The edge states exhibit a linear dispersion near the Fermi energy giving rise to a Dirac Fermion behavior, which can lead to an efficient transport of charge carriers. The Dirac Fermions are protected by time-reversal symmetry and hence are robust against nonmagnetic defects, disorder and surface contamination ${ }^{4-7}$. The QSH effect was first proposed by Kane and Mele for graphene, considering the opening of a band gap at the Dirac point by spin-orbit coupling (SOC) ${ }^{1}$. However, this proposal was shown to be unrealistic ${ }^{8,9}$, because the strength of SOC in graphene is extremely small. Subsequently, Beneviget et al. proposed that the quantum well semiconductor HgTe-CdTe would be a QSH insulator ${ }^{5}$, which was soon confirmed experimentally by observing ballistic edge channels ${ }^{3}$. Nevertheless, these quantum-well-based QSH insulators have a very small bulk band gap so that the QSH effect is observed only at low temperatures (below $10 \mathrm{~K})^{5}$. Besides, the $\mathrm{HgTe}$-CdTe quantum wells as well as their analogues ${ }^{10,11}$ have other serious limitations such as toxicity, difficulty of preparation, and incompatibility with current silicon-based electronic technology.

In searching for practical QSH insulators, two key ingredients are essential: (1) the materials must have a large bulk gap to ensure the realization of a QSH effect at room temperature, and (2) they must be compatible with the current silicon-based electronic technology. The latter condition narrows down our attention to the group IV honeycomb lattices. Silicene can be a nontrivial QSH insulator in its native structure, and can be observed in an experimentally accessible low temperature ${ }^{12}$. However, up to now, no free-standing silicene has been prepared experimentally, and its QSH effect can be observed only at low temperature ${ }^{13}$. Closely related to silicene in terms of compatibility and structure are germanene and stanene, which have been proposed as QSH insulators with a relative large band gap induced by spin-orbit coupling (SOC) ${ }^{12-15}$. However, they have not yet been realized experimentally. As a stable free-standing species that is experimentally accessible, only the germanane, $\mathrm{GeH}$, can be well produced $^{16,17}$. When strained, germanane can display a QSH effect ${ }^{18}$. However, it begins to become amorphous upon annealing at $75^{\circ} \mathrm{C}$ and becomes completely amorphous above $175^{\circ} \mathrm{C}^{16}$, which is undesirable because most transistors should operate up to hundreds of degrees ${ }^{\circ} \mathrm{C}$. Recently, a methyl-substituted germanane, $\mathrm{GeCH}_{3}$, has been synthesized $^{19}$ (Fig. 1a, b). In contrast to the case of germanane $\mathrm{GeH}$, it is found that $\mathrm{GeCH}_{3}$ has considerably enhanced thermal stability ${ }^{19}$. Therefore, it is worthwhile to probe whether $\mathrm{GeCH}_{3}$ has a QSH effect.

In this work, we study the electronic states of a single layer of $\mathrm{GeCH}_{3}$ by density functional calculations to show that reasonable strain could induce the s-p band inversion in the $\mathrm{GeCH}_{3}$ layer and makes it a QSH insulator, and that SOC plays an important role in opening a local band gap at the crossing points near the Fermi level. In the band-inverted $\mathrm{GeCH}_{3}$ layer under $12 \%$ strain, the band gap is $108 \mathrm{meV}$, so that this QSH insulator can be useful in an experimentally accessible temperature regime.

\section{Results}

Stability of $\mathrm{GeCH}_{3}$ monolayer. $\mathrm{GeCH}_{3}$ crystallizes in a hexagonal structure in which $\mathrm{GeCH}_{3}$ layers stack along the $c$ axis ${ }^{19}$. $\mathrm{GeCH}_{3}$ is thermally stable up to $250^{\circ} \mathrm{C}$, so it is quite promising as a building block for future 

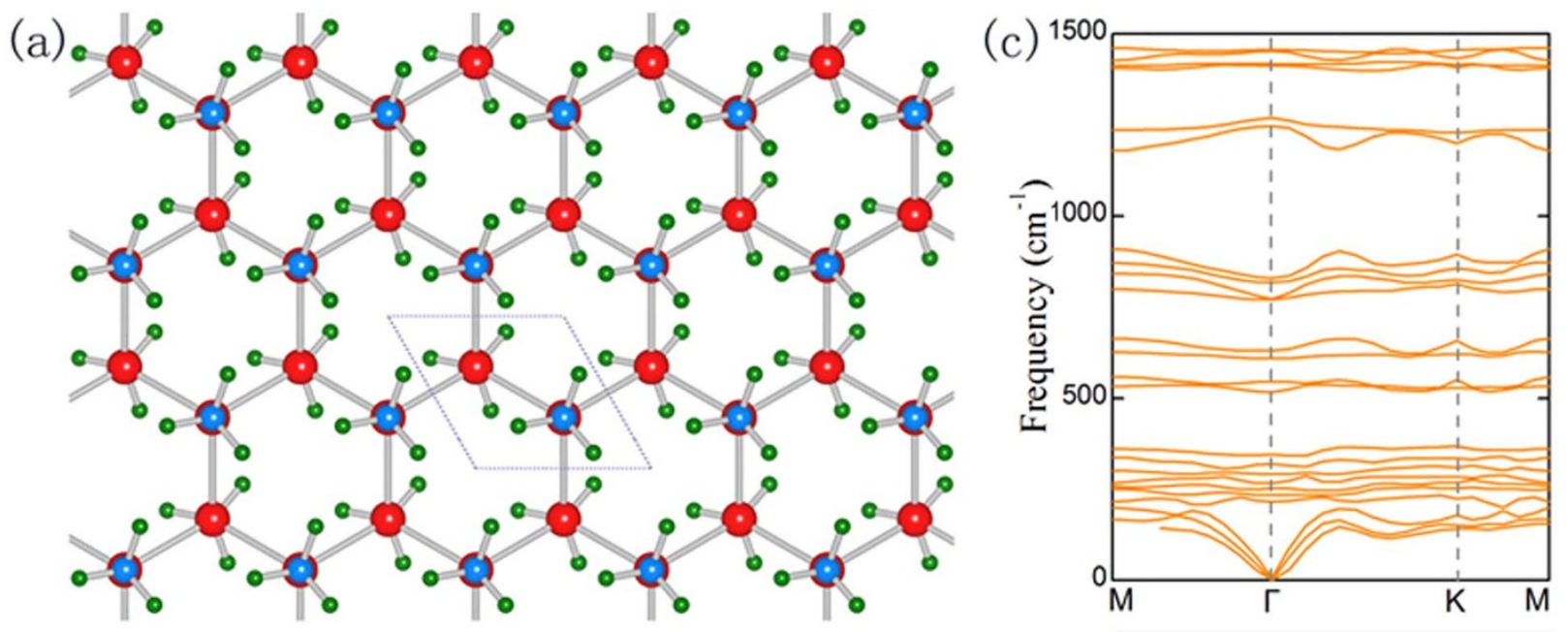

(b)

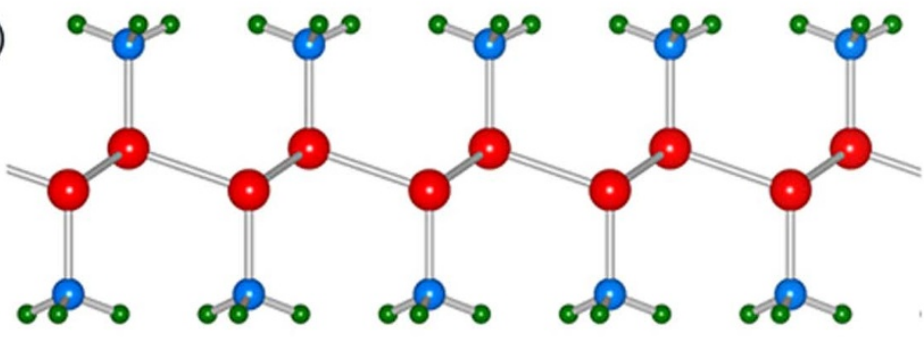

(d)

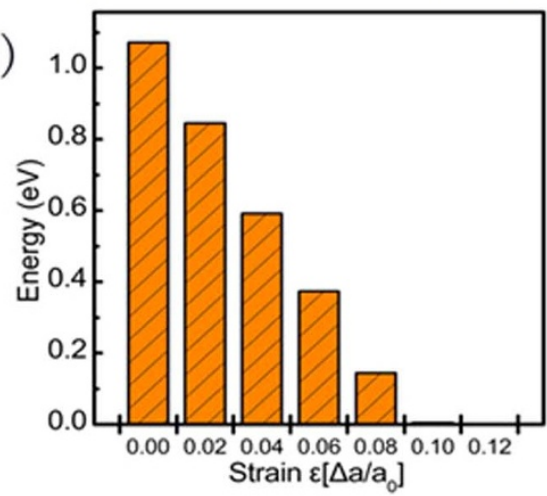

Figure 1 (a) Top and (b) side views of a $\mathrm{GeCH}_{3}$ monolayer. The red, blue and green balls denote $\mathrm{Ge}, \mathrm{C}$ and $\mathrm{H}$ atoms, respectively. (c) Phonon band dispersion relations calculated for a $\mathrm{GeCH}_{3}$ monolayer. (d) Energy gap calculated for a $\mathrm{GeCH}_{3}$ monolayer as a function of strain.

nanodevices ${ }^{15}$. Due to its large interlayer separation the electronic interaction between adjacent $\mathrm{GeCH}_{3}$ layers is extremely weak ${ }^{19}$, contrary to the case of graphite, which has a substantial interlayer overlap between the $\mathrm{C} 2 \mathrm{p}_{\mathrm{z}}$ orbitals, and $\mathrm{MoS}_{2}$ at the $\Gamma$ point, which has a substantial interlayer overlap between the $\mathrm{S} 3 \mathrm{p}_{\mathrm{z}}$ orbitals $^{20}$. The interlayer binding energy is calculated to be $5.7 \mathrm{meV} / \AA^{2}$ for $\mathrm{GeCH}_{3}$, which is even smaller than that of graphite $\left(9.3 \mathrm{meV} / \AA^{2}\right)$. Such weak coupling between $\mathrm{GeCH}_{3}$ layers provides a natural cleaving plane. Thus, bulk $\mathrm{GeCH}_{3}$ can be easily exfoliated to produce stable $\mathrm{GeCH}_{3}$ monolayers. The stability of $\mathrm{GeCH}_{3}$ monolayer is further confirmed by the phonon dispersion curves, presented in Fig. 1c, which shows that all branches of the phonon dispersion curves have positive frequencies, and no imaginary phonon modes are found. This confirms the stability of a $\mathrm{GeCH}_{3}$ monolayer.

Electronic structures of $\mathrm{GeCH}_{3}$ monolayer. As shown in Fig. 1a, b, each $\mathrm{sp}^{3}$-hybridized $\mathrm{Ge}$ atom is bonded to three Ge atoms and one $\mathrm{CH}_{3}$ group such that the buckled sheet of Ge atoms is sandwiched between two sheets of $\mathrm{CH}_{3}$ groups. Each $\mathrm{GeCH}_{3}$ monolayer has a hexagonal unit cell, and there are two Ge atoms and two $\mathrm{CH}_{3}$ groups per unit cell. In the optimized structure, the lattice constant is $3.954 \AA$ with $\mathrm{Ge}-\mathrm{Ge}=2.415 \AA$ and $\mathrm{Ge}-\mathrm{C}=1.972 \AA$.

The band structures calculated for a $\mathrm{GeCH}_{3}$ monolayer without and with SOC are plotted in Fig. 2. Without SOC, the band structure has a direct band gap $(1.07 \mathrm{eV})$ at the $\Gamma$ point (Fig. $2 \mathrm{a})$. The Ge- $4 \mathrm{~s}$ and Ge-4p states are located near Fermi level, with the Ge-4p states lying lower than the Ge-4s states. The valence band maximum (VBM) is doubly degenerate at $\Gamma$ because they are derived from the Ge- $4 \mathrm{p}_{\mathrm{x}, \mathrm{y}}$ states. When SOC is included, the degenerate level is split by a gap of $193 \mathrm{meV}$ (Fig. 2e, f). Except for this, no significant change is caused by SOC for the band structure of a $\mathrm{GeCH}_{3}$ mono- layer; the conduction band minimum (CBM) at $\Gamma$ is still occupied by the Ge-4s states while the two valence bands near the Fermi level at $\Gamma$ point are still dominated by Ge-4p states. Thus, no inverted band order is observed in this $2 \mathrm{D}$ lattice, showing that a $\mathrm{GeCH}_{3}$ monolayer is a trivial insulator.

Strain effects. Mechanical strain can induce the s-p band inversion and hence lead to a nontrivial topological state ${ }^{21-23}$. For example, under external strain, the layered compound InSe becomes a three dimensional $\mathrm{TI}^{22}$. In addition, strain can be readily exerted on nanosheets. Thus, one might consider if a $\mathrm{GeCH}_{3}$ monolayer becomes a QSH insulator under mechanical strain. We probe this question by introducing $0-12 \%$ strain into a $\mathrm{GeCH}_{3}$ monolayer maintaining all crystal symmetries and the overall honeycomb-like structure. Here we define the strain as $\varepsilon=\Delta a / a_{0}$, where $a_{0}$ and $a=$ $\Delta a+a_{0}$ are the lattice constants of the unstrained and strained cell, respectively. The $\mathrm{GeCH}_{3}$ lattice is expanded uniformly along both zigzag and armchair directions in the $a b$ plane and is relaxed along the $c$ axis. To clarify the stability of strained $\mathrm{GeCH}_{3}$, we also calculate phonon dispersion curves of $\mathrm{GeCH}_{3}$ under different strain. The corresponding results are shown in Fig. S1. We find that, the strain can reach to $12 \%$ without destroying the feature that imaginary phonon mode is absent in the phonon dispersion curves. This suggests that strained systems maintain their stability within the strain range of $12 \%$.

The evolution of the band gap of a $\mathrm{GeCH}_{3}$ monolayer as a function of the applied mechanical strain, presented in Fig. 1d, shows that with increasing the mechanical strain, the CBM is driven continuously to shift downward to the Fermi level thus significantly decreasing the energy gap. Throughout the changes under strain, it has a direct band gap with the VBM and CBM both located at the $\Gamma$ point. The band 

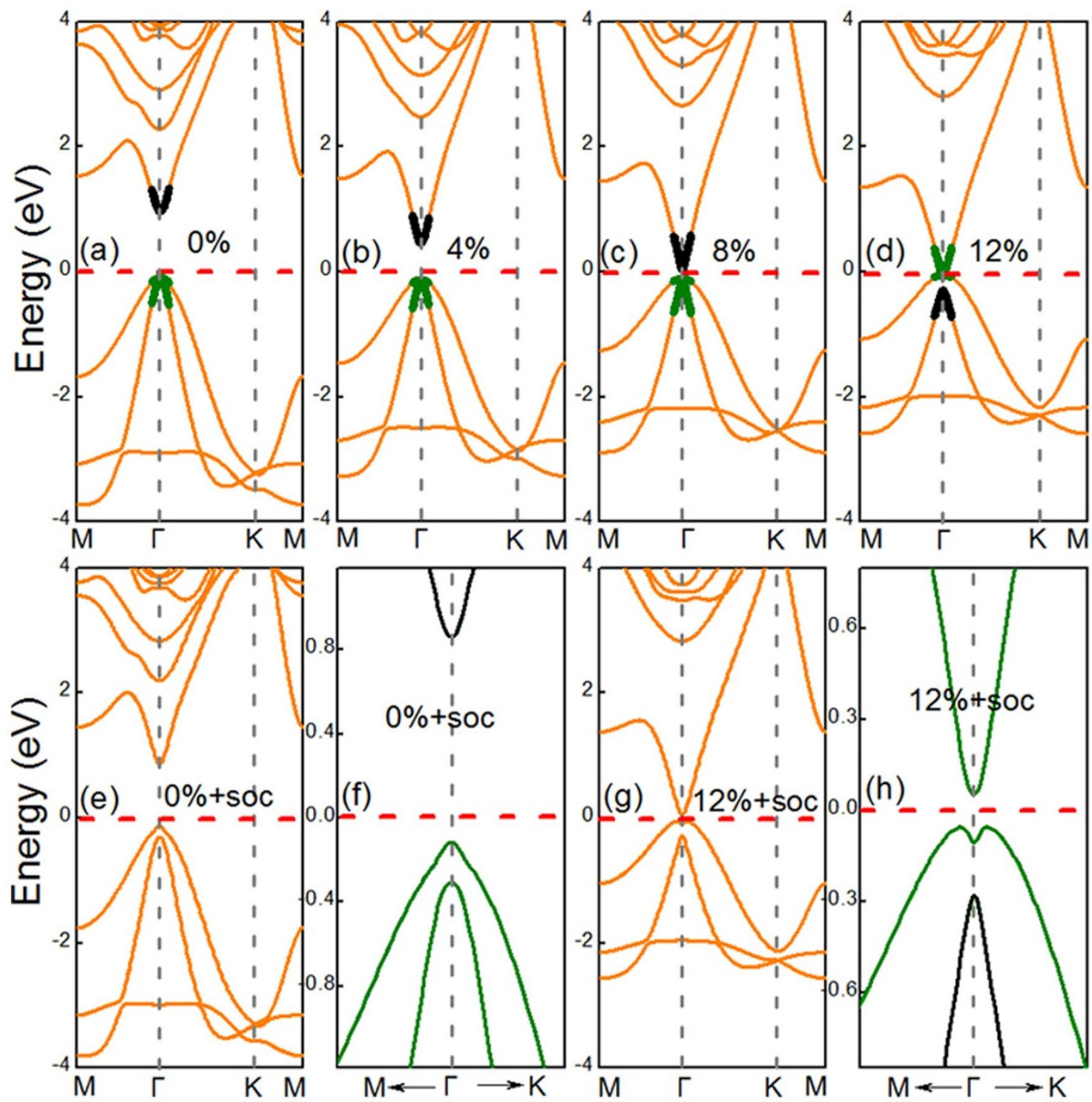

Figure $2 \mid$ The band structures calculated without SOC for an isolated $\mathrm{GeCH}_{3}$ monolayer structures with (a) $0 \%$, (b) $4 \%$, (c) $8 \%$ and (d) $12 \%$ strain. The band structures calculated with SOC for an isolated $\mathrm{GeCH}_{3}$ monolayer structures with (e) $0 \%$ and (g) $12 \%$ strain. (f) and (h) show zoomed-in views of (e) and (g), respectively. The black and olive lines denote the Ge-4s and Ge-4p orbitals, respectively. The horizontal dashed lines indicate the Fermi level.

structures of a $\mathrm{GeCH}_{3}$ monolayer under the strain of $4 \%, 8 \%$ and $12 \%$ are presented in Fig. 2b-d. From Fig. 2, we can see that, with $12 \%$ strain, the $\mathrm{GeCH}_{3}$ monolayer becomes a semimetal since the VBM and CBM is degenerate at $\Gamma$ point, and the s-p band inversion occurs around Fermi level; for the other two cases with strain below $\varepsilon=$ $12 \%$, the lowest-conduction band is represented by the Ge-4s states, and the highest two valence bands by the Ge-4p states. For the $\mathrm{GeCH}_{3}$ monolayer with $\varepsilon=12 \%$, the lowest-conduction band and the highest-valence band are represented by the Ge-4p orbitals, but the next-highest-valence band by the Ge-4s states. This establishes the existence of band inversion for the $\mathrm{GeCH}_{3}$ monolayer with $\varepsilon=$ $12 \%$. The inversion of band orders is a strong indication for the formation of nontrivial topological phases. The s-p band order inversion in our case is caused predominantly by strain, but the SOC is still indispensable because it lifts the band degeneracy at $\Gamma$, as shown in Fig. 2h. With SOC, a finite energy gap of $161 \mathrm{meV}$ opens at $\Gamma$ (an indirect gap of $108 \mathrm{meV}$ ) while the band order stays unchanged. This large SOC-induced nontrivial band gap reflects the fact that the states near the Fermi level are made up of $\sigma$ orbitals rather than $\pi$ orbitals ${ }^{18,24,25}$. The SOC-induced nontrivial energy gap is significantly greater than $\mathrm{k}_{\mathrm{B}} \mathrm{T}$ at room temperature $(=26 \mathrm{meV})$, so the QSH effect can be measured at room temperature. Thus $\mathrm{GeCH}_{3}$ might have useful applications in fields such as phase-change random access memory, quantum computing and spintronics.

The topological phase transition in a $\mathrm{GeCH}_{3}$ monolayer is further confirmed by calculating the $\mathrm{Z}_{2}$ topological invariant before and after the band inversion. Nontrivial topological insulators can be distinguished from normal insulators by nontrivial $Z_{2}$ topological invariant associated with the "bulk" electronic structure: $Z_{2}=1$ characterizes a nontrivial band topology, but $\mathrm{Z}_{2}=0$ a trivial band topology. All the structures of a $\mathrm{GeCH}_{3}$ monolayer considered here possess inversion symmetry. According to the method developed by $\mathrm{Fu}$ and $\mathrm{Kane}^{26}$, the existence of inversion symmetry allows us to calculate the $\mathrm{Z}_{2}$ topological invariant directly from knowledge about the parity of the Bloch wave functions for all the filled bands at all the time-reversal-invariant momenta (TRIM). For a $\mathrm{GeCH}_{3}$ monolayer, there are four TRIM points, namely, one $\Gamma$ point and three $M$ points in the Brillouin zone. The $\mathrm{Z}_{2}$ invariant is determined by the quantity $\delta_{\mathrm{i}}=\prod_{\mathrm{m}=1}^{\mathrm{N}} \xi_{2 \mathrm{~m}}\left(\mathrm{k}_{\mathrm{i}}\right)$, where $\xi_{2 \mathrm{~m}}\left(\mathrm{k}_{\mathrm{i}}\right)= \pm 1$ is the parity eigenvalue of the 
occupied band at the TRIM points $\mathrm{k}_{\mathrm{i}}$. The $\mathrm{Z}_{2}$ invariant is then given by the product of all the $\delta_{i}$ 's, $\prod_{i} \delta_{i}$. For the $\mathrm{GeCH}_{3}$ monolayer without strain or with strain below $8 \%$, they have trivial band topology with the topological invariant $Z_{2}=0$. When the strain is $12 \%$, the calculated $Z_{2}$ topological invariants is 1 , which confirms that the strain induces a topological phase transition.

The $2 \mathrm{D}$ nontrivial insulating states in the $\mathrm{GeCH}_{3}$ monolayer with $\varepsilon=12 \%$ should support an odd number of topologically protected Dirac-like edge states connecting the conduction and valence band edges at certain k-points. To see the nontrivial topological features of the $\mathrm{GeCH}_{3}$ monolayer with $\varepsilon=12 \%$ explicitly, we performed calculations of its edge-state band structure by constructing an zigzag nanoribbon structure with symmetric edges, for which we saturate the dangling bonds of all the edge atoms by hydrogen atoms. The width of the nanoribbon structure adopted here is $8.2 \mathrm{~nm}$, which is enough to separate the interaction between its two edges. The corresponding band structure of the nanoribbon plotted in Fig. 3a shows that the nontrivial topological gapless edge states connecting the band edge states and form a single Dirac cone at the $\Gamma$ point. For this nanoribbon structure, the two edges are the same, leading to two energetically degenerate Dirac cones located at the opposite edges. At a given edge, two counter-propagating edge states exhibit opposite spin-polarizations, a manifestation of the one-dimensional nature of the system. Thus, the surface-state calculation is consistent with the above parity analysis, and confirms conclusively the topologically non-trivial nature of this material.

Let us now consider how strain influences the band gap and the s-p band order. There are two factors affecting the band order; one is SOC, and the other is the strain. The latter factor has been discussed in many previous studies ${ }^{15,18,22,23,27}$. The previous showed that the band inversion in a $\mathrm{GeCH}_{3}$ monolayer can be driven by mechanical strain instead of SOC. Thus the physics of band inversion can be understood without considering SOC, while the effect of SOC is to create an energy gap afterwards. As discussed above, the valence and conduction bands away from the $\Gamma$ point are well separated, therefore, one needs to focus on the bands near the $\Gamma$ point. The relevant bands are dominated by the Ge- $4 \mathrm{~s}$ and $\mathrm{Ge}-4 \mathrm{p}_{\mathrm{x}, \mathrm{y}}$ states. Due to the chemical bonding between adjacent Ge atoms, these states are split into bonding and antibonding states, which we denote as $\left|s^{ \pm}\right\rangle$and $\left|p_{x, y}^{ \pm}\right\rangle$, with the subscript \pm representing the parity (Fig. 3b, c). For the $\mathrm{GeCH}_{3}$ monolayer without strain (Fig. 3b), the bands near the Fermi level are contributed by the $\left|s^{-}\right\rangle$and $\left|p_{x, y}^{+}\right\rangle$orbitals, with the $\left|s^{-}\right\rangle$orbital being above the $\left|p_{x, y}^{+}\right\rangle$orbital. For the $\mathrm{GeCH}_{3}$ monolayer with strain, increasing the lattice constant weakens the interaction between the Ge atoms. This decreases the splitting between the bonding and antibonding states, which lowers the $\left|s^{-}\right\rangle$state and raises $\left|p_{x, y}^{+}\right\rangle$state. Consequently, depending on the strength of strain, the energy gap of $\mathrm{GeCH}_{3}$ monolayer can be continuously tuned. At $12 \%$ strain, the $\left|p_{x, y}^{+}\right\rangle$level shifts above the $\left|s^{-}\right\rangle$level, which implies the normal band order is inverted under this condition, as depicted in Fig. 3c. Then the SOC then can lift the band degeneracy and create an energy gap at the $\Gamma$ point, signifying that a $\mathrm{GeCH}_{3}$ monolayer with $\varepsilon=12 \%$ is a $\mathrm{QSH}$ insulator.

\section{Discussion}

Our density functional calculations show that a topological phase transition can be induced in a recently synthesized $\mathrm{GeCH}_{3}$ monolayer by strain, and our phonon dispersion analysis shows that this layer is stable. The analysis of the $\mathrm{Z}_{2}$ topological invariant confirms the topological insulating nature of the insulating state of the $\mathrm{GeCH}_{3}$ monolayer with $12 \%$ strain. This QSH insulator has a large enough band gap that exceeds the thermal energy at room-temperature, making them suitable for room-temperature spintronics applications. It should be mentioned that there are many different ways to apply strain. For example, the strain can be realized by exerting mechanical forces to suspended samples, while very strong strain can also be introduced in thin films via the lattice mismatch with the substrate. For deposition onto substrates, one main concern is whether interface effect on the topological properties can be ignored.

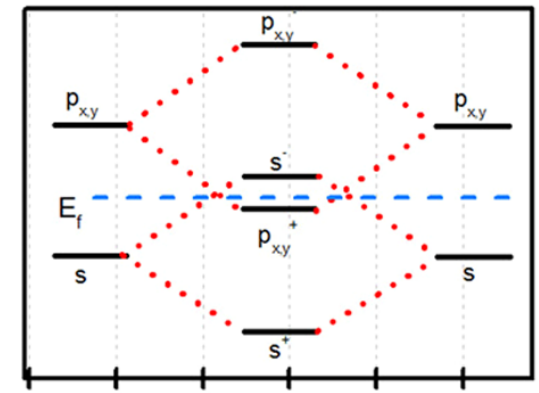

(b)

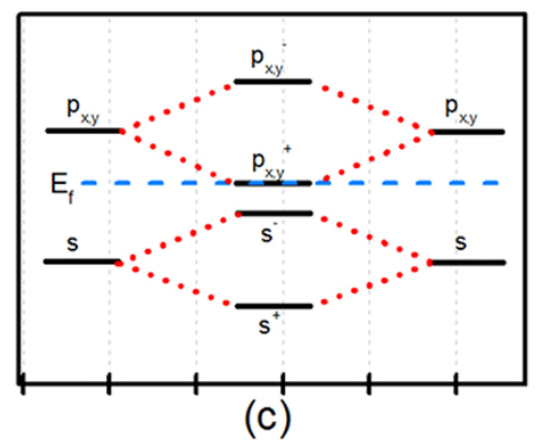

Figure $3 \mathrm{(a)} \mathrm{Edge} \mathrm{states} \mathrm{of} \mathrm{a} \mathrm{GeCH}_{3}$ monolayer calculated by using a nanoribbon model (see the text). Schematic diagrams showing the relative positions at $\Gamma$ of the Ge-4s and Ge-4p states of a $\mathrm{GeCH}_{3}$ monolayer near the Fermi level for the case of (b) $0 \%$ and (c) $12 \%$ strain in the absence of SOC. The horizontal dashed lines indicate the Fermi level. 
To this end, lots of efforts are still needed. However, as the first step for its realization, it is necessary to investigate the topological nature of free-standing $\mathrm{GeCH}_{3}$ as discussed in the present work. In actual conditions, there are many other factors that may affect the topological properties of $\mathrm{GeCH}_{3}$, so a more comprehensive study concerning these problems should be carried out in further works.

It is of interest to comment on the advantages of this $\mathrm{GeCH}_{3} \mathrm{QSH}$ insulator over other QSH insulators. First, the $\mathrm{GeCH}_{3}$ monolayer is made up of the commonly used elements and is thermally stable up to $250^{\circ} \mathrm{C}$, offering compatibility with current silicon-based electronic technology. Second, on the experimental side, $\mathrm{GeCH}_{3}$ has been synthesized whereas other 2D group IV lattice-based QSH insulators with comparable nontrivial gap (such as silicene, germanene, stanene, silicane, and stanane) have not yet been produced in the free-standing form. Third, the $\mathrm{CH}_{3}$ terminated surface can be resilient against oxidation, whereas the $-\mathrm{H}$ terminated surface (such as germanane and silicane) are extremely air sensitive. Finally, strain can switch between a normal insulating edge state and an almost linear dispersion for the metallicg edge state. Therefore, the $\mathrm{GeCH}_{3}$ monolayer allows one to construct a potential QSH field-effect transistor.

The peculiar judgment of the nontrivial topological nature is the existence of band inversion. Standard DFT calculations tend to underestimate the gap, and this could give an enhanced and not reliable estimate of the strain for band inversion. To overcome this drawback, we repeat the simulations using the Heyd-ScuseriaErnzerhof (HSE) functional ${ }^{28}$, and the corresponding results are plotted in Fig. S2. We find that the band inversion occurs at $13 \%$ strain based on HSE, which increases only a few percent with respect to the LDA case. From this we can argue that standard DFT is still a good approximation to study the band inversion feature in $\mathrm{GeCH}_{3}$ monolayer.

\section{Methods}

Our density functional calculations for an isolated $\mathrm{GeCH}_{3}$ layer were performed using the projector augmented wave $(\mathrm{PAW})$ method $^{29,30}$ implemented in the

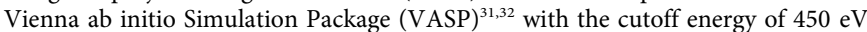
for the plane-wave basis set and the local density approximation (LDA $)^{33}$ to describe the exchange-correlation functional. Repeated image interactions were made negligible by including a vacuum layer of $18 \AA$ in the simulations. The Monkhorst-Pack set ${ }^{34}$ of $17 \times 17 \times 1$ k-points was used to sample the Brillouin zone for both geometry optimizations and total energy calculations. The lattice constants and the atom coordinates were fully optimized until the forces on each ion are reduced below $0.02 \mathrm{eV} / \mathrm{A}$. The optimization with the inclusion of van der Waals interactions are also tested, and the results show the main conclusions obtained here are not affected (see Fig. S3). Complementary calculations were performed to determine the phonon dispersion relations using the finite displacement method with the density functional perturbation theory (DFPT) as implemented in the CASTEP code $^{35,36}$

1. Kane, C. L. \& Mele, E. J. Quantum spin Hall effect in grapheme. Phys. Rev. Lett. 95, 226801 (2005)

2. Kane, C. L. \& Mele, E. J. $Z_{2}$ topological order and the quantum spin Hall effect. Phys. Rev. Lett. 95, 146802 (2005).

3. Bernevig, B. A. \& Zhang, S. C. Quantum spin Hall effect. Phys. Rev. Lett. 96, 106802 (2006).

4. Bernevig, B. A., Hughes, T. L. \& Zhang, S. C. Quantum spin Hall effect and topological phase transition in HgTe quantum wells. Science 314, 1757-1761 (2006).

5. Konig, M. et al. HgTe quantum wells. Science 318, 766-770 (2007).

6. Murakami, S. Quantum spin Hall effect and enhanced magnetic response by spinorbit coupling. Phys. Rev. Lett. 97, 236805 (2006).

7. Chuang, F.-C. et al. Tunable topological electronic structures in $\mathrm{Sb}(111)$ bilayers: A first-principles study. Appl. Phys. Lett. 102, 022424 (2013).

8. Yao, Y. G., Ye, F., Qi, X. L., Zhang, S. C. \& Fang, Z. Spin-orbit gap of graphene: First-principles calculations. Phys. Rev. B 75, 041401 (2007).

9. Huertas-Hernando, D., Guinea, F. \& Brataas, A. Spin-orbit coupling in curved graphene, fullerenes, nanotubes, and nanotube caps. Phys. Rev. B 74, 155426 (2006).

10. Liu, C., Hughes, T. L., Qi, X. L., Wang, K. \& Zhang, S. C. Quantum spin Hall effect in inverted type-II semiconductors. Phys. Rev. Lett. 100, 236601 (2008).
11. Knez, I., Du, R. \& Sullivan, G. Evidence for helical edge modes in inverted InAs/ GaSb quantum wells. Phys. Rev. Lett. 107, 136603 (2011).

12. Liu, C.-C., Feng, W. \& Yao, Y. Quantum spin Hall effect in silicene and twodimensional germanium. Phys. Rev. Lett. 107, 076802 (2011).

13. Novoselov, K. et al. A roadmap for grapheme. Nature 490, 192-220 (2012)

14. Liu, C.-C., Jiang, H. \& Yao, Y. Low-energy effective Hamiltonian involving spinorbit coupling in silicene and two-dimensional germanium and tin. Phys. Rev. B 84, 195430 (2011).

15. Xu, Y. et al. Large-gap quantum spin hall insulators in tin film. Phys. Rev. Lett. 111, 136804 (2013).

16. Bianco, E. et al. Stability and exfoliation of germanane: A germanium graphane analogue. ACS Nano 7, 4414-4421 (2013).

17. Koski, K. J. \& Cui, Y. The new skinny in two-dimensional nanomaterials. ACS Nano 7, 3739-3743 (2013).

18. Si, C. et al. Functionalized germanene as a prototype of large-gap two-dimensional topological insulators. Phys. Rev. B 89, 115429 (2014).

19. Jiang, S. S. et al. Improving the stability and optical properties of germanane via one-step covalent methyl-termination. Nat. Commun. 5, 3389 (2014).

20. Splendiani, A. et al. Emerging photoluminescence in monolayer $\mathrm{MoS}_{2}$. Nano Lett. 10, 1271-1275 (2010)

21. Zhao, L., Liu, J. W., Tang, P. Z. \& Duan, W. H. Design of strain-engineered quantum tunneling devices for topological surface states. Appl. Phys. Lett. 100, $131602(2012)$.

22. Ma, Y. D., Dai, Y., Yu, L., Niu, C. W. \& Huang, B. B. Engineering a topological phase transition in $\beta$-InSe via strain. New J. Phys. 15, 073008 (2013).

23. Feng, W. X. et al. Strain tuning of topological band order in cubic semiconductors. Phys. Rev. B 85, 195114 (2012).

24. Ma, Y. D., Dai, Y., Niu, C. W. \& Huang, B. B. Halogenated two-dimensional germanium: candidate materials for being of Quantum Spin Hall state. J. Mater. Chem. 22, 12587-12591 (2012).

25. Ma, Y. D., Dai, Y., Guo, M., Niu, C. W. \& Huang, B. B. Intriguing behavior of halogenated two-dimensional tin. J. Phys. Chem. C 116, 12977-12981 (2012).

26. Fu, L. \& Kane, C. L. Topological insulators with inversion symmetry. Phys. Rev. B 76, 045302 (2007).

27. Weng, H. M., Dai, X. \& Fang, Z. Transition-metal pentatelluride $\mathrm{ZrTe}_{5}$ and $\mathrm{HfTe}_{5}$ A paradigm for large-gap quantum spin Hall insulators. Phys. Rev. X 4, 011002 (2014).

28. Heyd, J., Scuseria, G. \& Ernzerhof, M. Heyd-Scuseria-Ernzerhof hybrid functional for calculating the lattice dynamics of semiconductors. J. Chem. Phys. 118, 8207 (2003).

29. Blöchl, P. E. Projector augmented-wave method. Phys. Rev. B 50, 17953.

30. Kresse, G. \& Joubert, D. From ultrasoft pseudopotentials to the projector augmented-wave method. Phys. Rev. B 59, 1758 (1999).

31. Kresse, G. \& Furthmüller, J. Efficiency of ab-initio total energy calculations for metals and semiconductors using a plane-wave basis set. Comput. Mater. Sci. 6 , 15-50 (1996).

32. Kresse, G. \& Furthmüller, J. Efficient iterative schemes for ab initio totalenergy calculations using a plane-wave basis set. Phys. Rev. B 54, 11169-11186 (1996).

33. Ceperley, D. M. \& Alder, B. J. Ground state of the electron gas by a stochastic method. Phys. Rev. Lett. 45, 566-569 (1980).

34. Monkhorst, H. J. \& Pack, J. D. Special points for Brillouin-zone integrations. Phys. Rev. B 13, 5188-5192 (1976).

35. Clark, S. J. et al. First principles methods using CASTEP. Z. Kristallogr. 220, 567-570 (2005)

36. Refson, K., Tulip, P. R. \& Clark, S. J. Variational density-functional perturbation theory for dielectrics and lattice dynamics. Phys. Rev. B 73, 155114 (2006).

\section{Acknowledgments}

This work is supported by the National Basic Research Program of China (973 program, 2013CB632401), National Science foundation of China under Grant 21333006, 11174180 and the Fund for Doctoral Program of National Education 20120131110066, 111 Project B13029.

\section{Author contributions}

Y.D. and B.B.H. designed the research. Y.D.M. and W.W. performed the density functional theory calculations. Y.D.M., Y.D. and M.-H.W. prepared the manuscript. All authors contributed to the scientific discussions.

\section{Additional information}

Supplementary information accompanies this paper at http://www.nature.com/ scientificreports

Competing financial interests: The authors declare no competing financial interests.

How to cite this article: Ma, Y., Dai, Y., Wei, W., Huang, B. \& Whangbo, M.-H.

Strain-induced quantum spin Hall effect in methyl-substituted germanane $\mathrm{GeCH}_{3}$. Sci. Rep. 4, 7297; DOI:10.1038/srep07297 (2014). 
(c) (i) $\Theta$ This work is licensed under a Creative Commons Attribution-NonCommercialNoDerivs 4.0 International License. The images or other third party material in this article are included in the article's Creative Commons license, unless indicated otherwise in the credit line; if the material is not included under the Creative
Commons license, users will need to obtain permission from the license holder in order to reproduce the material. To view a copy of this license, visit http:// creativecommons.org/licenses/by-nc-nd/4.0/ 